INVESTIGACIÓN

Recibido: 15/03/2020 --- Aceptado: 05/06/2020 --- Publicado: 12/03/2021

\title{
EVALUACIÓN DE LA IMPLEMENTACIÓN DE UN OBJETO DE APRENDIZAJE DESARROLLADO CON TECNOLOGÍA H5P
}

\section{Evaluation of the implementation of a learning object developed with H5P technology}

Sergio-Ramón Rossetti López¹. Universidad de Sonora. México. sergio.rossetti@unison.mx

Ma. Teresa García Ramírez. Universidad Autónoma de Querétaro. México. teregar@uaq.mx

(7) Isaac-Shamir Rojas Rodríguez. Universidad de Sonora. México. shamir.rojas@unison.mx

\section{RESUMEN}

Los objetos de aprendizaje son utilizados como materiales didácticos y tienen la finalidad de apoyar procesos de enseñanza y aprendizaje. El objetivo de este trabajo fue el de evaluar un objeto de aprendizaje, desarrollado con la herramienta de software libre H5P para ser utilizado como material de apoyo en un curso de Metodología de la Investigación en la Licenciatura en Administración en una universidad pública ubicada en el estado de Sonora, México. Con base en un estudio cuantitativo, descriptivo y transversal, se realizó la evaluación del objeto aprendizaje a partir de cuatro dimensiones: utilidad, impacto, integración y atributos. Los resultados obtenidos se derivan de una muestra de 70 estudiantes en donde se observa una valoración positiva del objeto de aprendizaje, además permiten vislumbrar el potencial y los beneficios que tiene la plataforma H5P para generar todo tipo de materiales educativos. Con los hallazgos de esta intervención, se dispone de un marco de referencia para futuros desarrollos e implementaciones de objetos de aprendizaje con H5P.

PALABRAS CLAVE: Evaluación - Objetos de aprendizaje - Tecnología educativa H5P - Sistema de gestión del aprendizaje.

\footnotetext{
1 Sergio Ramón - Rossetti López: Maestro en Gestión de Tecnologías de la Información, Ingeniero en Sistemas Computacionales. Departamento de Administración (Universidad de Sonora, México).
} 


\begin{abstract}
Learning objects are used as teaching materials and are intended to support teaching and learning processes. The objective of the article was to evaluate a learning object developed with the free software tool H5P to be used as support material in a course of Research Methodology in the Administration career at a public university located in the state of Sonora, Mexico. Based on a quantitative, descriptive and crosssectional study, the evaluation of the learning object was carried out on four dimensions: utility, impact, integration and characteristics. The results obtained are derived from a pilot with 70 undergraduate students and show a positive assessment of the learning object, they also allow us to glimpse the potential and benefits of the $\mathrm{H} 5 \mathrm{P}$ platform to generate all kinds of educational materials. With the findings of this intervention, a reference framework is available for future developments and implementations of learning objects with H5P.
\end{abstract}

KEY WORDS: Evaluation - Learning objects - Educational technology - H5P Learning management system.

\title{
AVALIAÇÃO DA IMPLEMENTAÇÃO DE UM OBJETO DE APRENDIZAGEM DESENVOLVIDO COM TECNOLOGIA H5P
}

\section{RESUMO}

Os objetos de aprendizagem são usados como materiais didáticos e tem a finalidade de apoiar os processos de ensino e aprendizagem. $\mathrm{O}$ objetivo deste trabalho foi avaliar um objeto de aprendizagem, desenvolvido com a ferramenta de software livre H5P para ser utilizado como material de apoio na matéria de Metodologia da pesquisa na Licenciatura em Administração em uma universidade pública, localizada no estado de Sonora, México. Baseado em um estudo quantitativo, descritivo e transversal, se fez a avaliação do objeto de aprendizagem a partir de quatro dimensões: utilidade, impacto, integração e atributos. Os resultados obtidos vem de uma amostra de 70 estudantes onde se observa uma valoração positiva do objeto de aprendizagem, além disso permite vislumbrar o potencial e os benefícios que tem a plataforma H5P para gerar todo tipo de materiais educativos. Com as descobertas desta intervenção, se abre um marco referencial para futuros desenvolvimentos e implementações de objetos de aprendizagem com H5P.

PALAVRAS CHAVE: Avaliação - objetos de aprendizagem - tecnologia educativa H5P - sistema de gestão do aprendizado.

\section{Cómo citar el artículo:}

Rossetti López, S. R., García Ramírez, M. T. y Rojas Rodríguez, I. S. (2021). Evaluación de la implementación de un objeto de aprendizaje desarrollado con tecnología H5P. Vivat Academia. Revista de Comunicación, 154, 1-24. http://doi.org/10.15178/va.2021.154.e1224. 
Rossetti López, S. R., García Ramírez, M. T. y Rojas Rodríguez, I. S.

Evaluación de la implementación de un objeto de aprendizaje desarrollado con tecnología H5P

http://www.vivatacademia.net/index.php/vivat/article/view/1224

\section{INTRODUCCIÓN}

Las Tecnologías de la Información y la Comunicación (TIC) en los últimos años, han sido consideradas como medios de soporte para las actividades educativas. La inclusión de tecnología en los procesos de enseñanza y aprendizaje ha confirmado su eficacia al promover la participación y colaboración en el salón de clase por parte de los estudiantes. En los sistemas educativos del futuro las y los educadores deberán construir actividades de aprendizaje que sean estimulantes y los individuos aprenderán cuando y donde quieran, a un ritmo con el que sientan cómodos, y valiéndose de las herramientas tecnológicas que estén a su alcance (Scott, 2015).

En este sentido, la incorporación de diferentes herramientas tecnológicas por parte de los docentes ha facilitado el proceso de adquisición de nuevos conocimientos en las poblaciones estudiantiles. Por lo anterior, contar con docentes capacitados y habilitados para el manejo de las TIC es de suma importancia para su correcta implementación en el aula (Del Moral, Martínez, y Neira, 2014).

Una de las herramientas que ha sobresalido en la educación mediada por tecnología, son los Sistemas de Gestión del Aprendizaje (SGA) (Duygu, Alkiş, y Ozkan-Yildirim, 2018), los cuales constituyen estructuras de vanguardia para el manejo de gran información dentro de los procesos de enseñanza aprendizaje (Cantabella, Martínez-España, Ayuso, Yáñez, y Muñoz, 2019). En el contexto mundial, en 2019, aproximadamente el 50\% de las clases universitarias estuvieron disponibles a través de un SGA, mientras que en México el 51\% de los estudiantes utilizan plataformas en línea para estudiar una carrera o un curso (AMIPCI, 2018).

Cada vez son más las instituciones educativas que utilizan los SGA como soporte en el proceso de enseñanza y aprendizaje en programas educativos (Concannon, Flynn, y Campbell, 2005; Lavigne, Backhoff-Escudero, y Organista-Sandoval, 2008). Estos sistemas actúan como facilitadores para llevar a cabo el proceso de formación a través de Internet y sirven de apoyo tanto para cursos totalmente a distancia, como mixtos y tradicionales de forma presencial.

La implementación de este tipo de tecnologías en el ámbito de la educación ha apoyado los procesos educativos, a la vez que han flexibilizado el aprendizaje en tiempo y espacio mediante las herramientas de comunicación y colaboración. Según autores como Baelo y Cantón (2010), las TIC se han convertido en un recurso que facilita el desarrollo de metodologías innovadoras en los procesos de enseñanza y aprendizaje. Sin embargo, para lograr el aprendizaje significativo mediante recursos de aprendizaje virtual es necesario que el estudiante se involucre y responsabilice de su aprendizaje y el profesor facilite al alumno los instrumentos de acceso a los recursos, que le permitan desarrollar, construir y explorar múltiples perspectivas o

Vivat Academia. Revista de Comunicación. 2021, nº 154, 1-24 
soluciones, favoreciendo así la inmersión del estudiante en un contexto que propicia el aprendizaje. Este cambio educativo hacia un modelo centrado en el alumno ha impulsado el desarrollo de los objetos de aprendizaje (OA) como apoyo a la docencia (Rosanigo, Bramati, López, Bramati, y Cotti, 2016). La demanda de plataformas y herramientas tecnológicas han propiciado la necesidad de desarrollar habilidades específicas en los profesores encargados de la elaboración de materiales digitales para ser incorporados en los SGA (Gasparetti, De Medio, Limongelli, Sciarrone, y Temperini, 2018). Un recurso idóneo para ser incorporado y utilizado a través de los SGA son los OA, los cuales son recursos digitales con cierto nivel de interactividad e independencia, que pueden ensamblarse y utilizarse en diferentes situaciones de enseñanza-aprendizaje (García, 2013).

De acuerdo con Barroso, Cabero, y Moreno (2016), los OA se presentan como materiales educativos de gran utilidad para mediar el aprendizaje de los estudiantes. Así mismo, Marzal, Calzado, y Ruvalcaba (2015) refieren que los OA permiten diseñar actividades prácticas en las que los usuarios tienen el control del avance de su aprendizaje. Por su parte, Cardeño, Muñoz, Ortiz, y Alzate (2017), afirman que este tipo de recursos pueden ayudar a promover el autoestudio, reforzar los conocimientos vistos en clase, motivar el aprendizaje y mejorar el rendimiento académico de los estudiantes. Además, los OA pueden despertar un alto grado de interés por los estudiantes y, por ende, un elevado grado de satisfacción formativa (Cabero, Llorente, y Gutiérrez, 2017).

Para el autor Wiley (2007) los OA son recursos digitales que pueden ser utilizados como soporte para llevar a cabo el proceso de aprendizaje y cuentan con ciertas características como son: un formato digital, un propósito pedagógico, contenido interactivo y son reutilizables. Por su parte los autores Mason, Weller, y Pegler (2003) los definen como "piezas digitales de material de aprendizaje que direcciona a un tema claramente identificable y que tiene el potencial de ser reutilizado en diferentes contextos" (p. 201). Otra definición indica que los OA son una entidad digital que puede ser utilizada, reutilizada y referenciada durante el aprendizaje apoyado con tecnología (IEEE, 2011). De acuerdo con Naharro, Bonet, Cáceres, Fargueta, y García (2007) deben contar con la capacidad de actualización y/o modificación; así mismo, los OA deben poder ser utilizados en contextos diferentes a los que fueron creados. Las principales características que tienen los OA son su reutilización, accesibilidad, interoperabilidad, durabilidad, flexibilidad, impacto, portabilidad, atributos digitales, granularidad, adaptabilidad y productividad (García, 2005; Herrera, Gelvez, y Sánchez, 2014; Vieira, De Morales, y Rossato, 2016).

En el contexto internacional, algunas instituciones y organismos han realizado esfuerzos para impulsar el desarrollo y difusión de OA como apoyo en la educación superior. En muchos casos, se han creado repositorios de OA que sirven como bancos de almacenamiento y distribución. Tal es el caso del MIT Open Coursware (OCW) en el cual se encuentran una gran cantidad de recursos educativos abiertos para estudiantes autónomos en el mundo. Otro repositorio es MERLOT (Multimedia Educational Resource for Learning and Online Teaching), el cual contiene bases de datos

Vivat Academia. Revista de Comunicación. 2021, nº 154, 1-24 
relativas a enseñanza digital y dispone de una gran cantidad de materiales pedagógicos de diversas disciplinas en formatos de vídeo, podcast, foros, OA y otros. Por su parte, Learning Resource Exchange (LRE) es un espacio para el intercambio de recursos de aprendizaje apoyado por la European Schoolnet que permite a las escuelas encontrar contenido educativo proveniente de muchos países.

En América Latina se tienen algunos repositorios activos de apoyo a la educación superior, tal es el caso de los repositorios de OA de la Universidad de Antioquia en Colombia, la Universidad de Guanajuato y el Instituto Tecnológico de Sonora, estos dos últimos en México. Todos estos esfuerzos se encuentran alineados a la política establecida por la Organización de las Naciones Unidas para la Educación, la Ciencia y la Cultura, la cual considera que a través de los Recursos Educativos Abiertos (REA) se ofrece una oportunidad estratégica de mejorar la calidad de la educación y el diálogo sobre políticas, el intercambio de conocimientos y el aumento de capacidades (UNESCO, 2015).

En este sentido, existen estudios que han obtenido evidencias acerca de los beneficios y mejoras en la calidad de la educación y el aprendizaje de los estudiantes al utilizar OA como apoyo en las clases (Angrist y Lavy, 2002; Carr, Taasoobshirazi, Stroud, y Royer, 2011). Sin embargo, en estos estudios se han utilizado OA desarrollados por especialistas en diseño multimedia y en otros casos, se utilizaron OA publicados en repositorios (Farha, 2009; Kay y Knaack, 2009; Lau y Woods, 2008; Navas y Cabero, 2007). Esto se debe principalmente a que, en años anteriores diseñar OA era una tarea más compleja para ser realizada por parte de los docentes, debido a que se requería conocimiento de tres áreas específicas. Por un lado, los conocimientos del experto en la materia encargado de seleccionar los contenidos de acuerdo con la planificación de curso, conocimientos sobre pedagogía para seleccionar el tipo de recurso y tecnología a utilizar, basado en diversos factores como el modelo educativo, diseño instruccional, tipo de contenidos, ente otros; y conocimientos de diseño multimedia para diseñar y ensamblar el objeto de aprendizaje.

Las herramientas tradicionales para la creación de OA están basadas en el modelo SCORM (Sharable Content Object Reference Model), y estas suelen ser técnicamente complejas para usuarios sin conocimientos especializados en diseño multimedia y, además, representan un costo importante para las instituciones que desean utilizarlas. En la tabla 1, se presentan algunas herramientas de software que actualmente se utilizan para desarrollar OA.

Tabla 1. Herramientas de software para crear OA.

\begin{tabular}{|c|c|c|c|c|}
\hline Herramienta & Tipo & Tipo de licencia & $\begin{array}{c}\text { Prueba } \\
\text { gratis }\end{array}$ & $\begin{array}{c}\text { Tipo de integración con } \\
\text { un LMS }\end{array}$ \\
\hline Elucidat & Authoring platform & Suscripción & SI & SCORM \\
\hline Adobe Captivate & Standalone authoring tool & Suscripción & SI & SCORM \\
\hline Gomo & Online authoring tool & Suscripción & SI & SCORM \\
\hline
\end{tabular}


Rossetti López, S. R., García Ramírez, M. T. y Rojas Rodríguez, I. S. Evaluación de la implementación de un objeto de aprendizaje desarrollado con tecnología H5P

\begin{tabular}{|c|c|c|c|c|}
\hline Lectora & Standalone authoring tool & Suscripción & SI & SCORM \\
\hline DominKnow & Online authoring tool & Suscripción & SI & SCORM \\
\hline Easygenerator & Online authoring tool & Suscripción & SI & SCORM \\
\hline iSpring Suite & Authoring suite & Suscripción & SI & SCORM \\
\hline H5P & Authoring platform & Libre & NO & Plugin, nativa y SCORM \\
\hline
\end{tabular}

Fuente: Elaboración propia, 2019.

Una gran ventaja y característica de la plataforma libre H5P radica en su código abierto y la posibilidad de crear, compartir y reutilizar contenido interactivo de una manera muy sencilla y sin costo. Esta herramienta se distribuye bajo el licenciamiento de tipo MIT (Massachusetts Institute of Technology) de software libre. El contenido generado en H5P es desarrollado en lenguaje HTML5 (HyperText Markup Language, versión 5) y es compatible con prácticamente cualquier dispositivo como computadoras, tabletas, celulares o laptops (Rekhari y Sinnayah, 2018; y Hsiung, 2018). Asimismo, H5P utiliza el estándar Experience API (xAPI) con el cual, es posible utilizar los OA en diferentes plataformas brindando la capacidad de almacenar el registro de las actividades de los usuarios con el recurso (Torrance y Wiggins, 2016). Lo anterior brinda mayor flexibilidad y portabilidad al contenido e-learning, a diferencia del modelo SCORM.

Para la implementación de OA el uso de herramientas emergentes como la plataforma $\mathrm{H} 5 \mathrm{P}$, redunda en mejoras en la presentación e interacción de los estudiantes con los contenidos y materiales de sus cursos, además facilitan la creación de contenido de aprendizaje interactivo por parte de los docentes de distintas áreas del conocimiento (Singh y Scholz, 2017). Además, H5P está basada en estándares de la W3C (World Wide Web Consortium) como la WCAG 2.0 (Web Content Accessibility Guideliness) la cual contempla una amplia gama de recomendaciones para crear contenido web con mejor accesibilidad.

Para crear OA mediante la herramienta H5P a través de un SGA, es necesario incorporar H5P a la plataforma educativa a través del plugin disponible en el portal www.hp5.org. Mediante esta opción, cualquier docente con privilegios puede agregar contenido $\mathrm{H} 5 \mathrm{P}$ como cualquier otro recurso o actividad nativa de la plataforma.

Por otro lado, es importante mencionar que la utilización de OA como estrategia de apoyo para el aprendizaje, está fundamentada en el enfoque constructivista (Baruque, Porto, y Melo, 2003), el cual describe al aprendizaje como un cambio significativo construido a partir experiencias. Una disyuntiva de los OA es desarrollar procesos de aprendizaje en los cuales se presente un marco conceptual sobre un tema en específico y se desarrollen actividades, problemas, casos o experiencias de aprendizaje que estimulen y adquieran significado para los estudiantes. Utilizar este tipo de recursos permite la construcción de escenarios en los que se desarrolla un aprendizaje activo de los estudiantes, los cuales participan y se 
convierten en agentes activos durante el proceso de aprendizaje (Christie y de Graaff, 2017), a través de la aproximación, interacción e involucramiento con los OA.

Es importante tomar en cuenta la calidad con la que se producen este tipo de recursos, para garantizar el propósito de su creación y que de alguna manera influyan positivamente en el aprendizaje que se está llevando a cabo, o de lo contrario, se correría el riesgo de que los estudiantes no los utilicen. Para efectos de esta investigación, se decidió seleccionar aquellos atributos de los OA que pudieran ser evaluadas desde la perspectiva del estudiante. Las características evaluadas en este estudio fueron utilidad, integración, impacto en el aprendizaje y los atributos. Además, dadas las características de la universidad intervenida, se utilizó el SGA Moodle como medio de distribución del objeto de aprendizaje.

\section{OBJETIVOS}

El objetivo general que se pretende con este estudio es evaluar la implementación un objeto de aprendizaje utilizado por estudiantes de administración en una universidad pública ubicada en el estado de Sonora, México. Los objetivos particulares que se buscan alcanzar son los siguientes.

1. Utilizar un modelo de diseño instruccional para crear un objeto de aprendizaje.

2. Desarrollar e implementar un objeto de aprendizaje como estrategia de aprendizaje activo bajo el enfoque constructivista.

3. Evaluar desde la perspectiva de los estudiantes universitarios cuatro dimensiones: utilidad, integración, impacto en el aprendizaje y atributos.

\section{METODOLOGÍA}

\subsection{Método}

Para llevar a cabo la investigación se utilizó un diseño no experimental con enfoque cuantitativo de corte transversal, mediante una metodología de tipo descriptiva y su propósito fue evaluar desde la perspectiva de los estudiantes un OA para el curso de Metodología de la Investigación que se imparte a estudiantes de octavo semestre de la carrera de Licenciatura en Administración (LA).

\subsection{Instrumento}

Para llevar a cabo la evaluación del OA desde la perspectiva de los estudiantes, se utilizó el cuestionario de evaluación de objetos de aprendizaje, creado por Centre of Excellence in Teaching y Learning (CETL) in Reusable Learning Objects (RLO). Este cuestionario permite evaluar utilidad, impacto en el aprendizaje, integración y atributos de un OA, a través de una escala de Likert y preguntas abiertas (Williams, O'Connor, Windle, y Wharrad, 2015). Para su aplicación, se diseñó un formulario web, el cual contenía los reactivos del cuestionario. 


\subsection{Población y muestra}

Esta investigación se realizó en la asignatura de Metodología de la Investigación en el ciclo escolar 2019-1. El muestreo fue no probabilístico utilizando el método por conveniencia. La muestra estuvo integrada por 70 estudiantes divididos en dos grupos, 10 hombres y 23 mujeres en el grupo 1; y 13 hombres y 24 mujeres del grupo 2.

\subsection{Diseño instruccional}

La creación de OA requiere de metodologías de diseño instruccional. El diseño instruccional se puede definir como un proceso en el cual se define y concreta de manera específica cómo tienen que ser y cómo deben relacionarse todos los elementos que integran una acción formativa (Stephenson y Sangrá, 2008).

El OA se desarrolló siguiendo el modelo de diseño instruccional ADDIE el cual incluye las fases de análisis, diseño, desarrollo, implementación y evaluación (Baruque et al., 2003). Este modelo ha sido uno de los más utilizados para el desarrollo de programas, cursos, materiales y entrenamientos. En este sentido, el docente de la materia intervenida recibió una capacitación del modelo ADDIE y de la herramienta H5P. En la figura 1, se muestran cada una de las fases y las actividades que se fueron desarrollando en el modelo ADDIE.

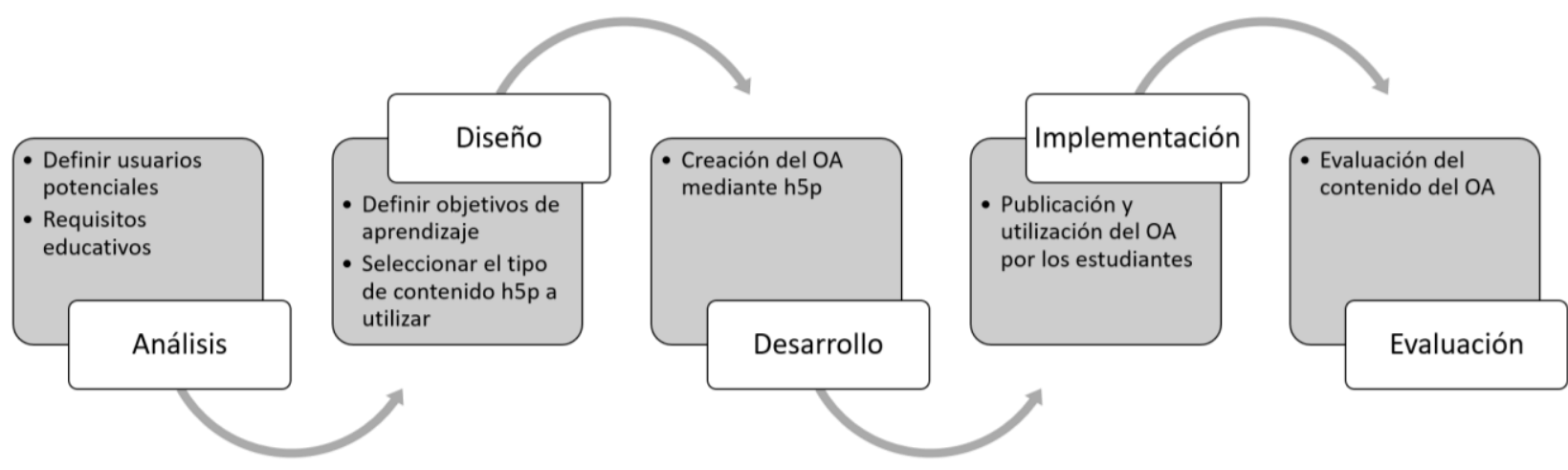

Figura 1. Aplicación del modelo ADDIE.

Fuente: Elaboración propia, 2019.

Es importante mencionar que la temática sobre la cual se diseñó el OA fue "alcances de investigación", tema propuesto por el docente de la materia debido a la problemática que han presentado estudiantes en cursos anteriores, para poder identificar con claridad los diferentes tipos de alances que puede tener una investigación científica.

Análisis: en esta fase se establecieron parámetros como las características de los estudiantes, carrera, semestre y asignaturas que en un momento dado se pudieran 
beneficiar con el OA. Con lo anterior se obtuvieron las metas instruccionales y la lista de las tareas a enseñarse.

También se logró identificar y conocer a los usuarios potenciales del OA teniendo las siguientes características: estudiantes inscritos a la materia de Metodología de la Investigación, bajo la modalidad de enseñanza presencial. En cuanto al perfil de los estudiantes, estos contaban con una edad en el rango de 22 a 27 años, pueden o no poseer conocimientos previos del tema alcances de investigación; y necesitan tener conocimientos básicos en el manejo de la computadora, para utilizar de mejor manera el OA.

En la tabla 2 se presenta el punto de vita de los estudiantes sobre su manejo de la computadora. En donde se observa que el $75.71 \%$ de los estudiantes inscritos a la materia, consideran tener un alto manejo de la computadora, el 12.6\% considera tener un nivel medio y el $11.43 \%$ un nivel bajo.

Tabla 2. Manejo de la computadora.

\begin{tabular}{|c|c|c|c|c|}
\hline Género & Bajo & Medio & Alto & Total \\
\hline Hombre & $7.14 \%$ & $5.71 \%$ & $20.00 \%$ & $32.86 \%$ \\
\hline Mujer & $4.29 \%$ & $7.14 \%$ & $55.71 \%$ & $67.14 \%$ \\
\hline Total & $11.43 \%$ & $12.86 \%$ & $75.71 \%$ & $100.00 \%$ \\
\hline
\end{tabular}

Fuente: Elaboración propia, 2019.

En la tabla 3, se muestra una comparativo entre la media de edad de los estudiantes y el grado de manejo de la computadora. En este sentido, los estudiantes con mayor edad consideran tener un nivel alto en el manejo de la computadora, mientras que los estudiantes de menor edad consideran tener un bajo nivel de uso.

Tabla 3. Manejo de la computadora.

\begin{tabular}{|c|c|c|c|c|}
\hline Género & Bajo & Medio & Alto & Total \\
\hline Hombre & 22.50 & 23.21 & 22.60 & 22.96 \\
\hline Mujer & 22.40 & 22.87 & 24.33 & 22.91 \\
\hline Total & 22.44 & 22.96 & 23.25 & 22.93 \\
\hline
\end{tabular}

Fuente: Elaboración propia, 2019.

Diseño: en esta fase se realizó la elaboración de un guion tecnopedagógico que ayudó a definir la interfaz del OA, la descripción del proceso de enseñanza aprendizaje a llevar a cabo, de acuerdo con el contexto, población objetivo, necesidades, recursos, medios de consulta, contenidos, objetivos, entre otros. En la figura 2, se muestra la estructura del guion tecnopedagógico utilizado, basado en Morales, Gutiérrez, y Ariza (2016). 
Rossetti López, S. R., García Ramírez, M. T. y Rojas Rodríguez, I. S.

Evaluación de la implementación de un objeto de aprendizaje desarrollado con tecnología H5P
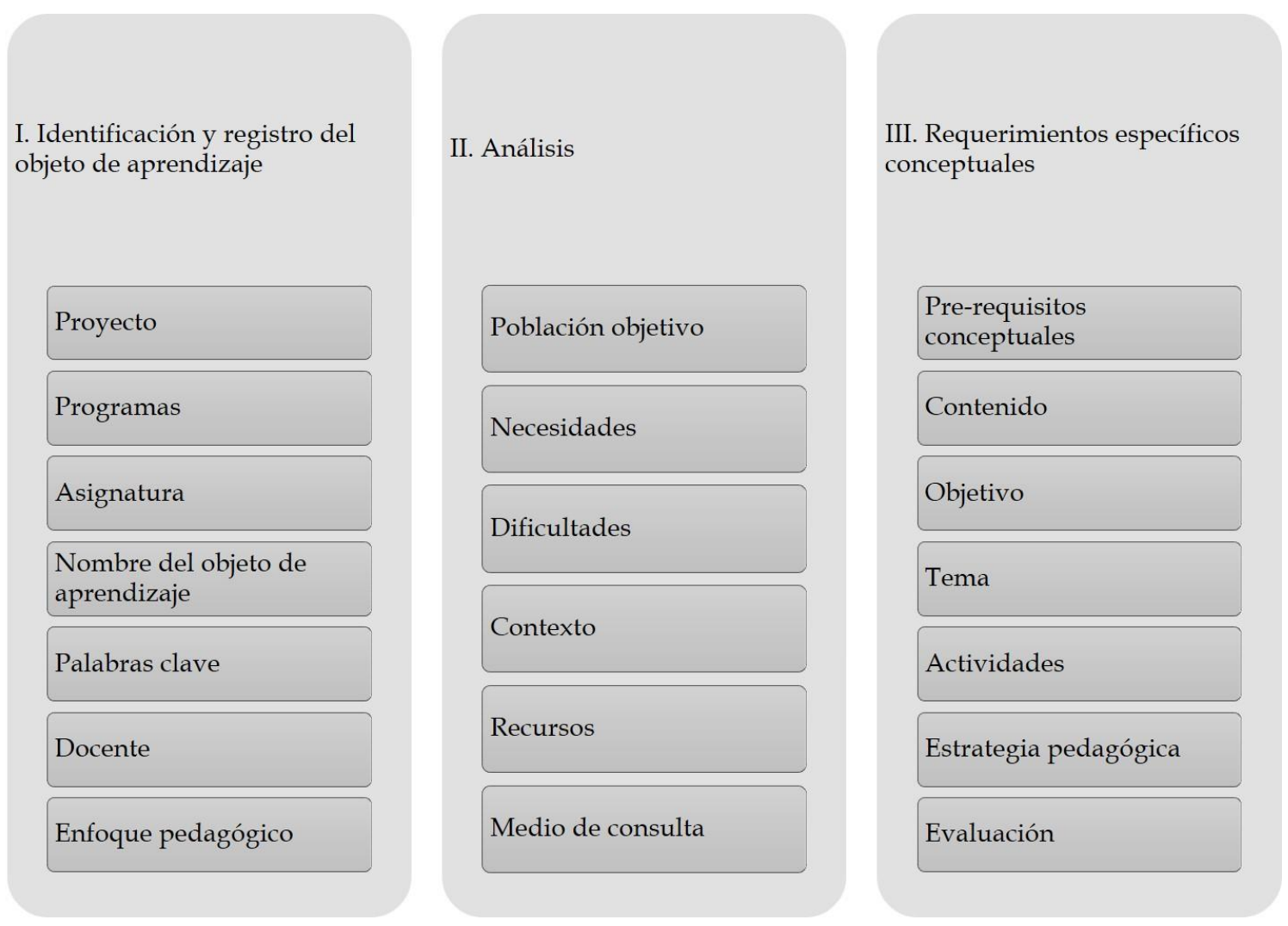

Figura 2. Estructura del guion tecnopedagógico.

Fuente: Elaboración propia, 2019.

Así mismo, se realizó un análisis de los contenidos y referencias bibliográficas para el diseño el OA. Se estableció el objetivo del tema, el cual era identificar los diferentes tipos de alcances que puede tener en una investigación. Para ello, se incluyó la presentación del tema, información relacionada a los conceptos teóricos, los cuales incluían la definición y clasificación de los alcances de una investigación, un video explicativo, actividades de aprendizaje que incluían preguntas de falso/verdadero y una sección para relacionar conceptos en la cual se ejemplificaban diferentes investigaciones y el estudiante debía seleccionar el tipo de alcance. Finalmente se incluyeron todas las referencias bibliográficas del tema. En la figura 3, se presenta la maquetación del OA. 
Rossetti López, S. R., García Ramírez, M. T. y Rojas Rodríguez, I. S. Evaluación de la implementación de un objeto de aprendizaje desarrollado con tecnología H5P

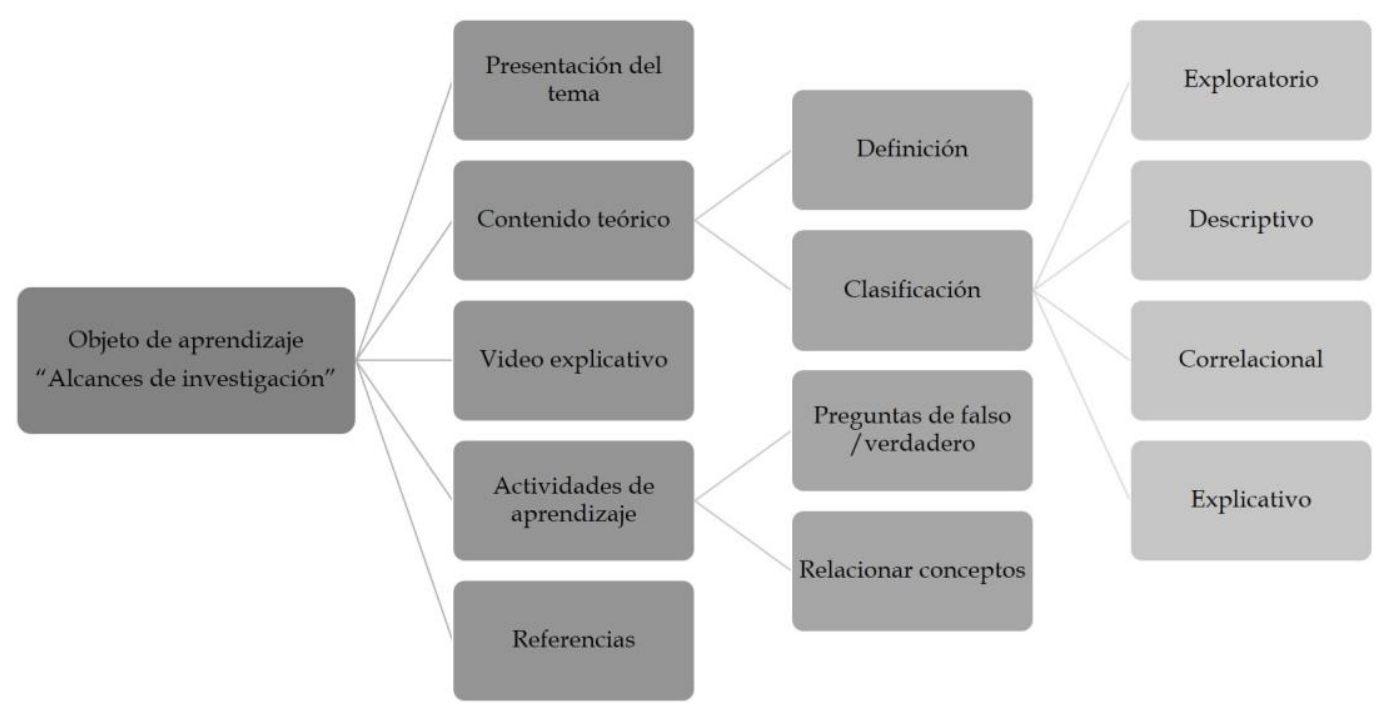

Figura 3. Maquetación del OA.

Fuente: Elaboración propia, 2019.

Desarrollo: para el desarrollo del OA se capacitó al docente de la materia en el uso de la plataforma H5P. Una vez desarrollado el OA, el docente programó la fecha y hora en qué estaría disponible el recurso en su aula virtual. En la figura 4, se observa una de las actividades del OA.

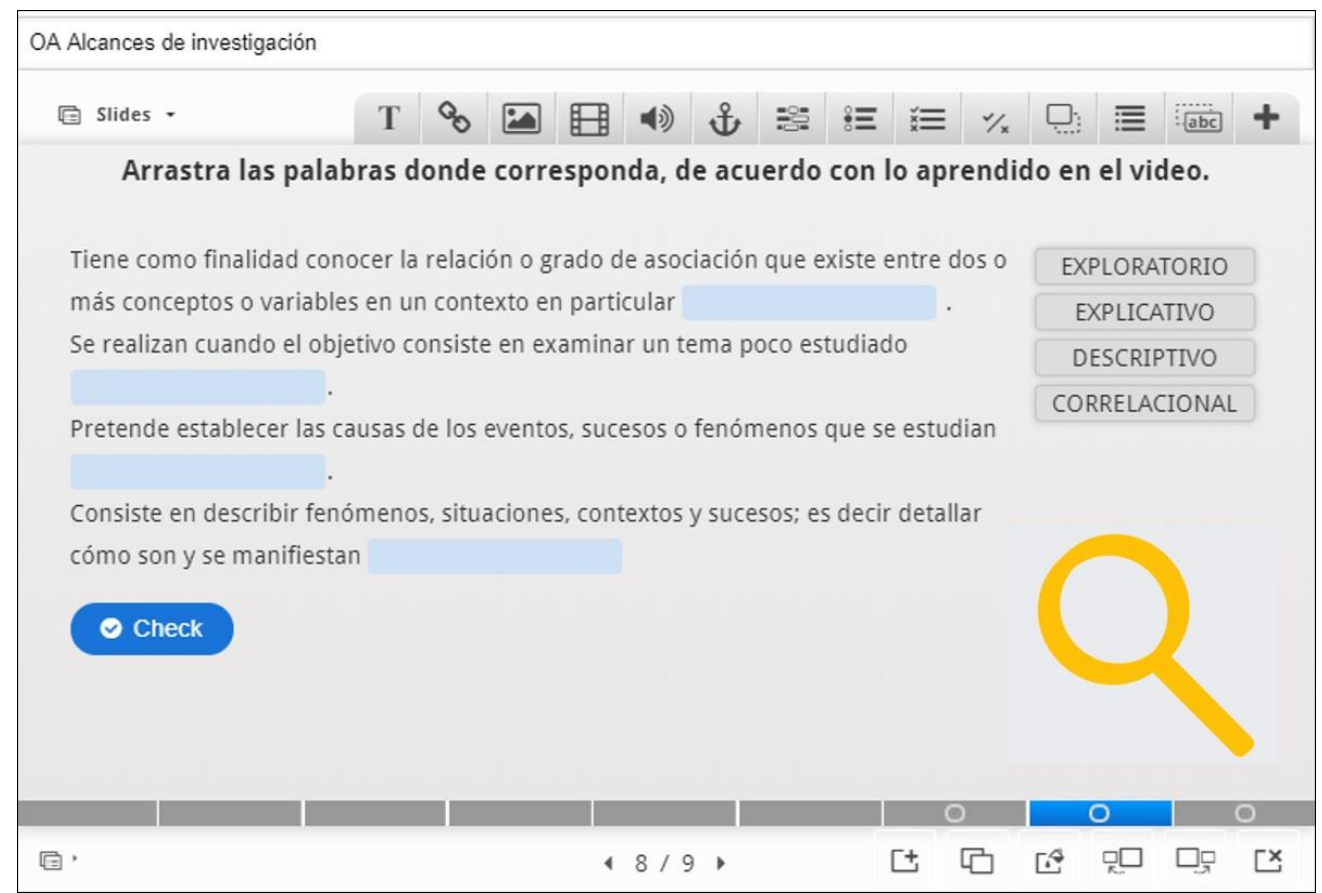

Figura 4. Diseño de actividad relacionar conceptos.

Fuente: Elaboración propia, 2019.

Implementación: el OA fue expuesto a un pilotaje en el ciclo 2019-1 (enero - julio) a la muestra de estudiantes. Los estudiantes tuvieron acceso a este recurso dentro del 
aula virtual que comúnmente utilizan como apoyo para sus clases. Inicialmente el docente aplicó un pre-test diagnóstico que contenía diez preguntas de opción múltiple sobre el tema. Posteriormente, presentó el objeto de aprendizaje "alcances de investigación". Una vez finalizado el tiempo de utilización del objeto de aprendizaje, se solicitó a los estudiantes que realizaran el post-test que contenía las mismas diez preguntas de la prueba diagnóstico

Evaluación: Se llevó a cabo la evaluación del objeto de aprendizaje acorde con las cuatro dimensiones anteriormente mencionadas, para ello se solicitó a los estudiantes abrir el vínculo que llevaba el cuestionario web. En el apartado de resultados se muestran los hallazgos más importantes.

\section{RESULTADOS}

En este apartado se presentan los resultados más relevantes sobre la evaluación del objeto de aprendizaje desde las dimensiones de utilidad, integración, impacto en el aprendizaje y atributos del objeto. Una de las preguntas iniciales del cuestionario tuvo el objetivo de identificar si el estudiante presentó algún tipo de problema técnico al utilizar el objeto de aprendizaje.

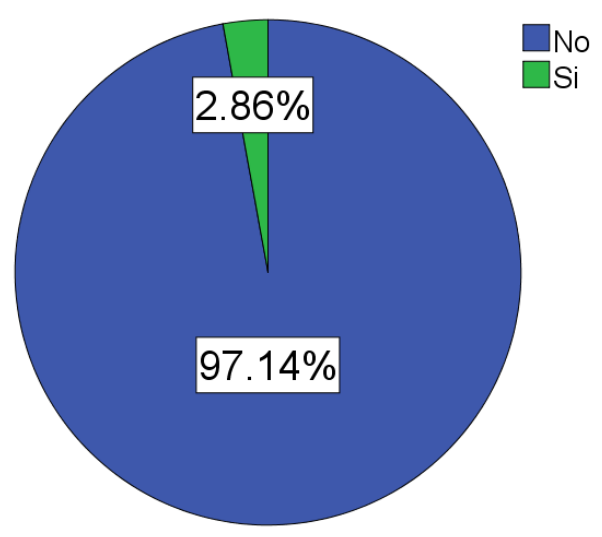

Figura 5. Problemática en el manejo del objeto de aprendizaje.

Fuente: Elaboración propia, 2019.

En la figura 5 se observa que el $97.14 \%$ de los estudiantes no tuvo problemas al utilizar el objeto de aprendizaje, sin embargo, se presentaron algunos inconvenientes como: "tardó en cargar el video del objeto de aprendizaje" y "no lograba encontrar el vínculo de acceso al objeto de aprendizaje". Es importante mencionar que estas problemáticas son ajenas al contenido y programación del objeto de aprendizaje. Sin embargo, son de tomar en consideración para el diseño instruccional de las actividades por parte del docente.

Mediante el uso de tablas de distribución de frecuencias porcentuales, se presentan los resultados obtenidos en cada una de las categorías. En la tabla 4, se muestran los resultados de la categoría "utilidad del objeto de aprendizaje", en 
donde desde la perspectiva de los estudiantes fue de utilidad, sin embargo, se requiere revisar los ejercicios de autoevaluación ya que un $12 \%$ considera que no les ayudó en su aprendizaje. Este resultado en particular brinda un área de oportunidad para mejorar las actividades incluidas en el objeto de aprendizaje. Así mismo, los ítems mejor evaluados de esta dimensión fueron la facilidad de navegación del objeto de aprendizaje con un $99 \%$ de respuestas positivas y los conceptos y lenguajes claros empleados en el objeto de aprendizaje con un 100\% de respuestas positivas.

Tabla 4. Utilidad del objeto de aprendizaje.

\begin{tabular}{|c|c|c|c|c|}
\hline ITEM & $\begin{array}{c}\text { Muy de } \\
\text { acuerdo }\end{array}$ & De acuerdo & Desacuerdo & $\begin{array}{c}\text { Muy en } \\
\text { desacuerdo }\end{array}$ \\
\hline $\begin{array}{c}\text { Los objetivos y propósito del } \\
\text { objeto de aprendizaje fueron } \\
\text { claros }\end{array}$ & $41 \%$ & $59 \%$ & $0 \%$ & $0 \%$ \\
\hline $\begin{array}{c}\text { El objeto de aprendizaje era } \\
\text { fácil de navegar }\end{array}$ & $53 \%$ & $46 \%$ & $1 \%$ & $0 \%$ \\
\hline $\begin{array}{c}\text { El objeto de aprendizaje tiene } \\
\text { conceptos y lenguaje claros }\end{array}$ & $49 \%$ & $51 \%$ & $0 \%$ & $0 \%$ \\
\hline $\begin{array}{c}\text { Ayudó a mi aprendizaje gracias } \\
\text { a que pude trabajar a mi propio } \\
\text { ritmo }\end{array}$ & $63 \%$ & $31 \%$ & $6 \%$ & $0 \%$ \\
\hline $\begin{array}{c}\text { Ayudó a mi aprendizaje gracias } \\
\text { a la interactividad }\end{array}$ & $61 \%$ & $35 \%$ & $4 \%$ & $1 \%$ \\
\hline $\begin{array}{c}\text { Ayudó a mi aprendizaje gracias } \\
\text { a los ejercicios de } \\
\text { autoevaluación }\end{array}$ & $47 \%$ & $47 \%$ & $6 \%$ & $0 \%$ \\
\hline $\begin{array}{c}\text { El objeto de aprendizaje tenía el } \\
\text { nivel académico adecuado }\end{array}$ & $47 \%$ & $41 \%$ & $0 \%$ & $0 \%$ \\
\hline
\end{tabular}

Fuente: Elaboración propia, 2019.

En la tabla 5, se muestran ahora los resultados sobre la categoría de integración del objeto de aprendizaje, en donde se observa que un 99\% están en algún grado de acuerdo sobre la inclusión de los OA en otros cursos. Así mismo, el 94\% consideran que el objeto de aprendizaje está bien relacionado con los temas del curso.

Tabla 5. Integración del objeto de aprendizaje.

\begin{tabular}{|c|c|c|c|c|}
\hline ITEM & $\begin{array}{c}\text { Muy de } \\
\text { acuerdo }\end{array}$ & De acuerdo & Desacuerdo & $\begin{array}{c}\text { Muy en } \\
\text { desacuerdo }\end{array}$ \\
\hline $\begin{array}{c}\text { Recomendaría el uso de este } \\
\text { objeto de aprendizaje a otros } \\
\text { estudiantes que lleven este }\end{array}$ & $53 \%$ & $41 \%$ & $6 \%$ & $0 \%$ \\
\hline
\end{tabular}


Rossetti López, S. R., García Ramírez, M. T. y Rojas Rodríguez, I. S. Evaluación de la implementación de un objeto de aprendizaje desarrollado con tecnología H5P

\begin{tabular}{|c|c|c|c|c|}
\hline curso & . & & \\
\hline $\begin{array}{c}\text { Me gustaría utilizar más objetos } \\
\text { de aprendizajes en otros } \\
\text { cursos }\end{array}$ & $43 \%$ & $56 \%$ & $1 \%$ & $0 \%$ \\
\hline $\begin{array}{c}\text { El contenido del objeto de } \\
\text { aprendizaje fue apropiado para } \\
\text { este curso }\end{array}$ & $50 \%$ & $44 \%$ & $6 \%$ & $0 \%$ \\
\hline $\begin{array}{c}\text { El objeto de aprendizaje está } \\
\text { bien relacionado con los temas } \\
\text { del curso }\end{array}$ & $53 \%$ & $44 \%$ & $3 \%$ & $0 \%$ \\
\hline
\end{tabular}

Fuente: Elaboración propia, 2019.

En la tabla 6, se presentan los resultados de la categoría relacionada con el impacto en el aprendizaje. Se observa una valoración muy positiva de la categoría, sin embargo, el 11\% de los usuarios considera que no les ayudó a entender el tema y un $7 \%$ que no contribuyó en el cumplimiento del programa de la materia.

Tabla 6. Impacto en el aprendizaje.

\begin{tabular}{|c|c|c|c|c|}
\hline ITEM & $\begin{array}{c}\text { Muy de } \\
\text { acuerdo }\end{array}$ & De acuerdo & Desacuerdo & $\begin{array}{c}\text { Muy en } \\
\text { desacuerdo }\end{array}$ \\
\hline $\begin{array}{c}\text { El objeto de aprendizaje me } \\
\text { ayudó a cumplir con el } \\
\text { programa de la materia }\end{array}$ & $63 \%$ & $30 \%$ & $7 \%$ & $0 \%$ \\
\hline $\begin{array}{c}\text { El objeto de aprendizaje me } \\
\text { ayudó a comprender un tema } \\
\text { específico sobre en el curso }\end{array}$ & $49 \%$ & $40 \%$ & $11 \%$ & $0 \%$ \\
\hline $\begin{array}{c}\text { El objeto de aprendizaje me } \\
\text { sirvió para retener información } \\
\text { sobre el tema }\end{array}$ & $59 \%$ & $39 \%$ & $3 \%$ & $0 \%$ \\
\hline
\end{tabular}

Fuente: Elaboración propia, 2019.

A continuación, en la tabla 7, se presenta la última categoría evaluada por parte de los estudiantes en esta investigación, la cual tiene que ver con los atributos propios del objeto de aprendizaje. Se observa que un 95\% de los estudiantes están de acuerdo o muy de acuerdo en los atributos visuales, de audio y de texto incluidos en el objeto de aprendizaje. Por otro lado, un 13\% mencionó que tuvo dificultades en el acceso al objeto de aprendizaje.

Tabla 7. Atributos del objeto de aprendizaje.

\begin{tabular}{|c|c|c|c|c|}
\hline ITEM & $\begin{array}{l}\text { Muy de } \\
\text { acuerdo }\end{array}$ & De acuerdo & Desacuerdo & $\begin{array}{c}\text { Muy en } \\
\text { desacuerdo }\end{array}$ \\
\hline
\end{tabular}


Rossetti López, S. R., García Ramírez, M. T. y Rojas Rodríguez, I. S. Evaluación de la implementación de un objeto de aprendizaje desarrollado con tecnología H5P

\begin{tabular}{|c|c|c|c|c|}
\hline $\begin{array}{c}\text { Componentes visuales (video, } \\
\text { imágenes, animaciones, etc.) }\end{array}$ & $44 \%$ & $51 \%$ & $5 \%$ & $0 \%$ \\
\hline Componentes de audio o texto & $61 \%$ & $34 \%$ & $5 \%$ & $0 \%$ \\
\hline Acceso en cualquier momento & $53 \%$ & $34 \%$ & $13 \%$ & $0 \%$ \\
\hline
\end{tabular}

Fuente: Elaboración propia, 2019.

En general, se obtuvieron valoraciones positivas en las cuatro categorías de evaluación del objeto de aprendizaje. Con el objetivo de identificar la categoría mejor evaluada, se agruparon las respuestas en positivas, aquellas con selección muy de acuerdo y de acuerdo; y en negativas, aquellas con selección en muy desacuerdo y en desacuerdo. En la figura 6, se presentan los resultados globales por categoría. Es de llamar la atención que la categoría que presenta un área de oportunidad para realizar mejoras al recurso es la relacionada a los atributos imagen, video y texto, la cual, presenta un $8 \%$ de respuestas negativas por parte de los estudiantes. Asimismo, la categoría mejor evaluada fue integración con un $96 \%$ de respuestas positivas.

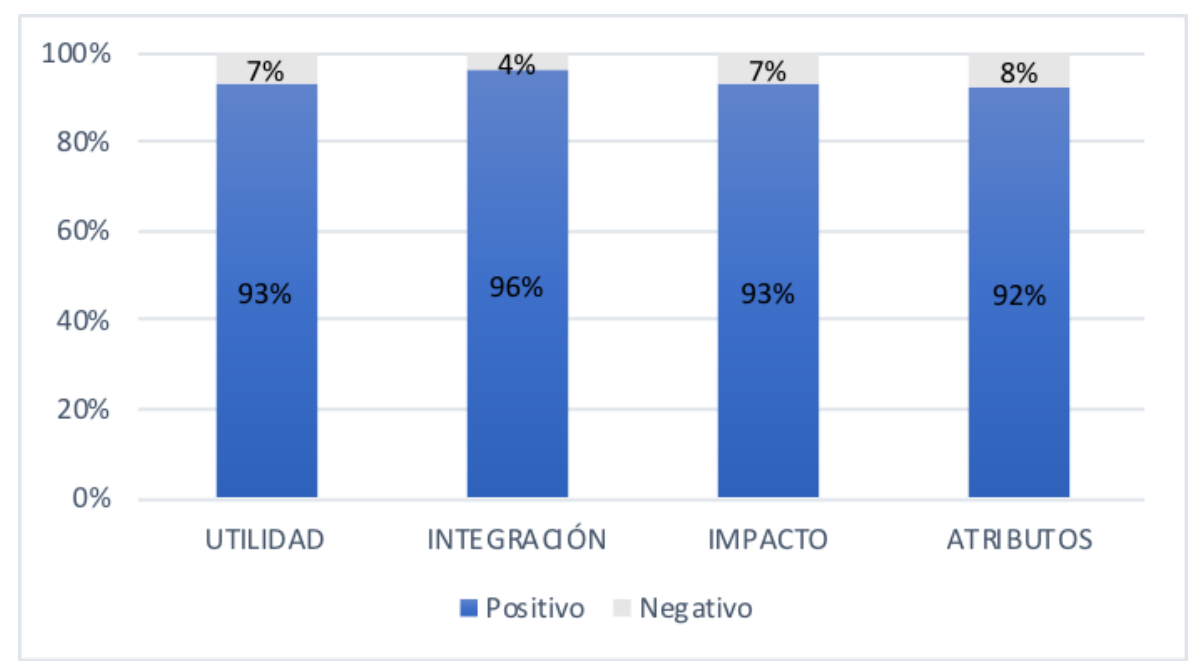

Figura 6. Resultados globales por categoría.

Fuente: Elaboración propia, 2019.

En la parte final de la evaluación del objeto de aprendizaje, los estudiantes respondieron dos preguntas abiertas. Utilizar este tipo de preguntas permite no ejercer influencia en la respuesta de los sujetos, abre el abanico de posibilidades de respuestas. Para realizar un análisis a estas preguntas, primero se identificaron las respuestas de mayor tendencia por parte de los sujetos. Posteriormente, las respuestas fueron codificadas y agrupadas de acuerdo con los siguientes criterios.

1. Observar la frecuencia con que aparece cada respuesta en la pregunta. 
2. Seleccionar las respuestas que se presentan con mayor frecuencia.

3. Clasificar las respuestas elegidas en tópicos o rubros, de acuerdo con un criterio lógico, cuidando que sean mutuamente excluyentes.

4. Asignar un nombre o título a cada rubro.

5. Asignar un código a cada patrón general de respuesta.

6. Eliminar aquellos patrones que tienen un bajo número de frecuencia.

Al realizar el conteo de palabras a las respuestas de las dos preguntas abiertas mediante el software ATLAS.ti, fue posible conocer que varias categorías tenían únicamente dos frecuencias. Por ello, éstos se redujeron a cuatro categorías por pregunta. La primera de las preguntas fue ¿cuáles consideran que serían los beneficios para el estudiante, el poder contar con más objetos de aprendizaje en clase? En la tabla 8 se muestran las categorías encontradas para la pregunta "sobre los beneficios de contar con más objetos de aprendizaje". Se puede observar que estas categorías se encuentran asociadas principalmente al "impacto en el aprendizaje".

Tabla 8. Beneficios para el estudiante.

\begin{tabular}{|c|c|}
\hline Categorías & Frecuencias \\
\hline Tener mayor conocimiento de un tema & 17 \\
\hline Tener información disponible sobre el tema & 17 \\
\hline Herramienta para mejorar el aprendizaje & 15 \\
\hline Comprender mejor un tema & 6 \\
\hline TOTAL & 55 \\
\hline
\end{tabular}

Fuente: Elaboración propia, 2019.

Haciendo uso de la misma herramienta de análisis, se obtuvieron los resultados a la segunda pregunta abierta del cuestionario, la cual fue: ¿cuáles consideran que serían los beneficios para el profesor, el poder contar con más objetos de aprendizaje en clase? En la tabla 9 se muestran los resultados de las categorías encontradas en el análisis Estas categorías permean en el "proceso de enseñanza" ya que consideran que el objeto de aprendizaje permite explicar de mejor manera un tema y con ello reforzar sus conocimientos.

Tabla 9. Beneficios para el profesor.

\begin{tabular}{|c|c|}
\hline Categorías & Frecuencias \\
\hline Explicar mejor un tema & 19 \\
\hline Facilitar enseñanza & 12 \\
\hline Mayor participación de los estudiantes & 14 \\
\hline Reforzar los conocimientos & 11 \\
\hline TOTAL & 56 \\
\hline
\end{tabular}

Fuente: Elaboración propia, 2019. 
En la figura 7 se muestra un diagrama de los beneficios percibidos por los estudiantes universitarios y los autores que corroboran estos hallazgos en otras investigaciones.

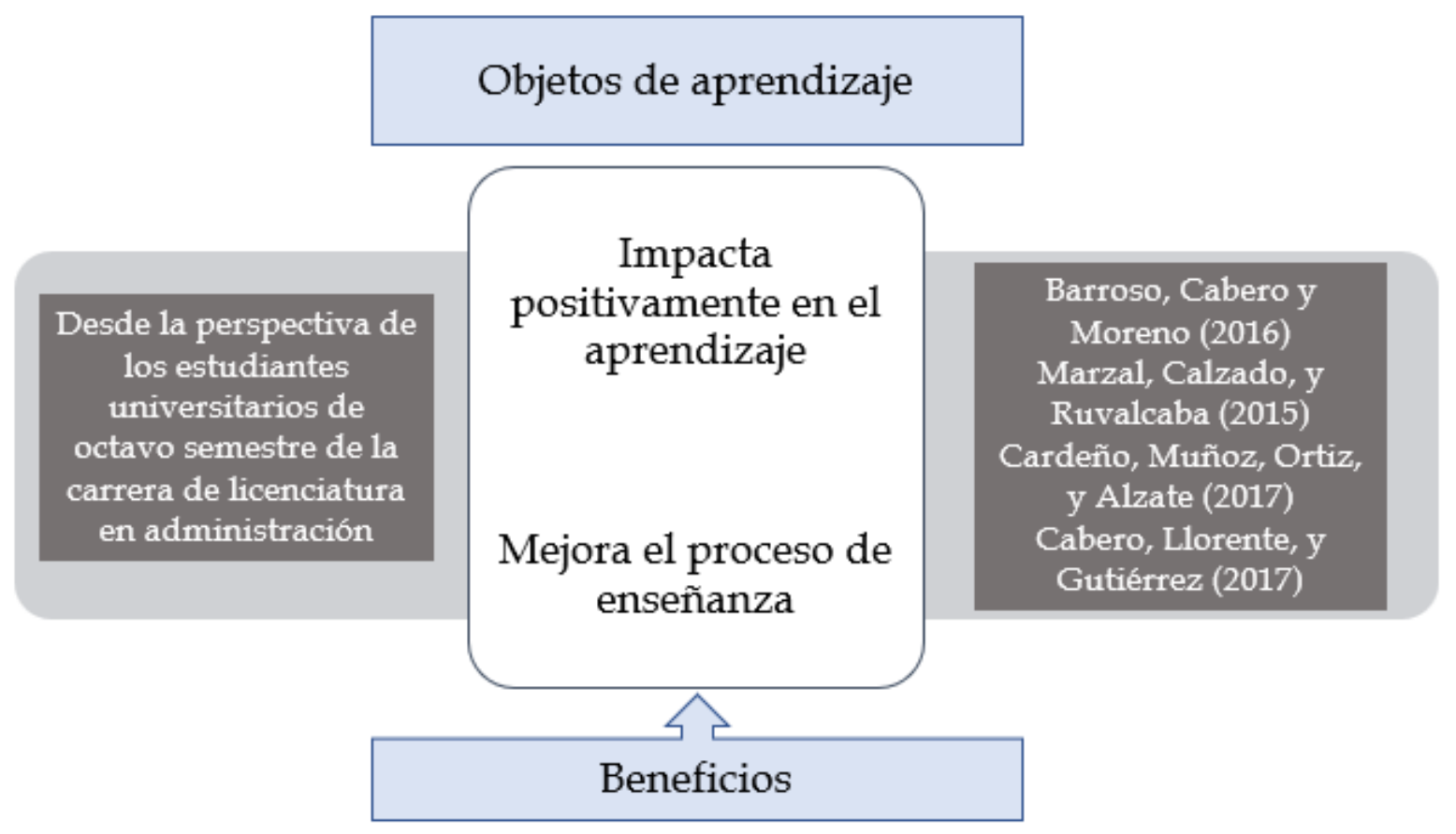

Figura 7. Beneficios percibidos de los OA.

Fuente: Elaboración propia, 2019.

\section{DISCUSIÓN}

Después de realizar el análisis de los resultados en la presente investigación, se revelaron algunos aspectos importantes relacionados con los objetivos que se plantearon. El modelo de diseño instruccional ADDIE permitió diseñar y desarrollar de manera ordenada el contenido y actividades del objeto de aprendizaje, lo cual concuerda con lo presentado por (García, 2015; y Díaz, Schiavoni, y Amadeo, 2011).

Los estadísticos descriptivos presentados en resultados ponen de manifiesto que los estudiantes que intervinieron en el estudio recomendarían la inclusión de más OA. Así mismo, los estudiantes percibieron al objeto de aprendizaje como fácil de utilizar y con objetivos muy claros. Por otro lado, también consideran como característica importante del objeto de aprendizaje, el poder trabajar a su ritmo, es decir, poder visitar el recurso, avanzar y retroceder en su contenido e incluso concluirlo o visitarlo de nuevo. Un área de oportunidad encontrada en el estudio tiene que ver con los ejercicios de autoevaluación incluidos en el objeto de aprendizaje, ya que el $12 \%$ de los estudiantes consideraron que estos no fueron de ayuda para el aprendizaje. Esto representa un reto para futuros docentes que deseen 
desarrollar este tipo de materiales y que puedan construir actividades de autoevaluación más estimulantes y significativas para los estudiantes.

Por otro lado, el $7 \%$ de los estudiantes que consideraron que el objeto de aprendizaje no contribuyó en el cumplimiento del programa, lo cual tiene que ver primordialmente con dos razones, el hecho de que el docente decidió no asignar una calificación al objeto de aprendizaje y que una de las limitantes del estudio fue que el tema abordado en el objeto de aprendizaje fue relativamente corto, lo que no motivó su uso por un periodo más largo. Será decisión de los docentes el determinar si este tipo de actividades se utilizan únicamente como reforzamiento y/o material de auto estudio, o bien, como una actividad que forme parte de la calificación final del curso.

El 13\% de los estudiantes que manifestaron tener algún tipo de dificultad en el acceso al objeto de aprendizaje, tuvieron problemas debido a que no recordaban su usuario y/o contraseña de acceso al SGA. Una alternativa de solución a este tipo de problemáticas es el publicar los OA como Recursos Educativos Abiertos dentro de un repositorio institucional, tal y como lo vienen haciendo algunas instituciones de Europa, Estados Unidos y América Latina.

Los resultados anteriores muestran que se puede implementar la estrategia presentada para crear $\mathrm{OA}$ en todos los cursos que se imparten en el programa de Administración de la universidad y con ello contar con un repositorio que esté disponible para los estudiantes de todos los semestres. Lo que impactaría en el aprendizaje activo y se promovería el autoaprendizaje de los estudiantes. Así mismo, la estrategia de implementación, evaluación y herramienta presentada en este trabajo podría ser utilizada por otras instituciones para la producción de sus OA.

Entre los comentarios mencionados por los estudiantes en las preguntas abiertas destacan; mejorar el aprendizaje, comprender mejor un tema, facilitar la enseñanza y reforzar conocimientos, lo que concuerda con Cardeño, Muñoz, Ortiz, y Alzate (2017), que mencionan que los OA pueden ayudar a promover el autoestudio, reforzar los conocimientos vistos en clase, motivar el aprendizaje y mejorar el rendimiento académico de los estudiantes. Además, de un elevado grado de satisfacción formativa (Cabero, Llorente, y Gutiérrez, 2017).

\section{CONCLUSIONES}

Para el caso particular de la institución educativa y la población de estudiantes intervenidos, el OA obtuvo en general valoraciones positivas, de acuerdo con el instrumento de cuestionario de evaluación de OA. En el cual, sobresalen del resto las respuestas a las preguntas relacionadas a facilidad de navegación, recomendación del objeto de aprendizaje y la relación del objeto con los temas del curso. Cabe señalar que H5P es compatible con Moodle y otras plataformas educativas, esta característica hace que H5P sobresalga de otros competidores, debido a que los usuarios no tienen que migrar sus cursos a otros ambientes de aprendizaje, es decir, pueden continuar con sus cursos alojados en el SGA de su preferencia e ir 
incorporando OA, sin afectar la estructura y recursos previos dentro del curso. Lo anterior abre un amplio abanico de opciones para los profesores, sin importar el nivel de estudios, debido a la posibilidad de poder crear comunidades virtuales para el intercambio de este tipo de contenidos.

Por otro lado, sobresalen algunos atributos del objeto de aprendizaje que fueron considerados por la población de estudio como muy importantes. Estos atributos son interactividad, componentes visuales como imágenes, audio y video, acceso en cualquier momento y trabajar a mi ritmo. Lo anterior indica que la población de estudio tiene preferencia por utilizar contenidos visuales, interactivos, dinámicos y accesibles desde cualquier lugar y en cualquier momento. Con el surgimiento de plataformas y tecnologías emergentes como H5P, los docentes ahora pueden desarrollar e implementar sus propios OA, debido fundamentalmente a la gran facilidad y operatividad que tienen las nuevas herramientas de software, las cuales no requieren de conocimientos de algún lenguaje especializado en programación de computadoras para crear materiales digitales atractivos e innovadores. Para el desarrollo del objeto de aprendizaje la herramienta H5P resultó una buena solución ya que es fácil de utilizar y no requiere de conocimientos de programación.

\section{REFERENCIAS}

AMIPCI. (2018). Asociación Mexicana de Internet. Educación en línea en México. Recuperado de https://www.asociaciondeinternet.mx/estudios

Angrist, J., \& Lavy, V. (2002). New evidence on classroom computers and pupil learning. Economic Journal, 112(482), 735-765. https://doi.org/10.1111/1468$\underline{0297.00068}$

Baelo, R., \& Cantón, I. (2010). Use of Information and Communication Technologies in Castilla \&amp; León Universities. Comunicar, 18(35), 159-166. https://doi.org/10.3916/C35-2010-03-09

Barroso, J., Cabero, J., \& Moreno, A. M. (2016). La utilización de objetos de aprendizaje en Realidad Aumentada en la enseñanza de la medicina. Innoeduca. International Journal of Technology and Educational Innovation, 2(2), 77. https://doi.org/10.20548/innoeduca.2016.v2i2.1955

Baruque, L. B., Porto, F., \& Melo, R. N. (2003). Towards an instructional design methodology based on learning objects. Proceedings of the IASTED International Conference on Computers and Advanced Technology in Education, 259-264.

Cabero, J., Llorente, C., \& Gutiérrez, J. J. (2017). Evaluación por y desde los usuarios: objetos de aprendizaje con Realidad aumentada. Revista de Educación a Distancia (RED), (53). https://doi.org/10.6018/red/53/4 
Cantabella, M., Martínez-España, R., Ayuso, B., Yáñez, J. A., \& Muñoz, A. (2019). Analysis of student behavior in learning management systems through a Big Data framework. Future Generation Computer Systems, 90, 262-272. https://doi.org/10.1016/j.future.2018.08.003

Cardeño, J., Muñoz, L. G., Ortiz, H. D., \& Alzate, N. C. (2017). La incidencia de los Objetos de Aprendizaje interactivos en el aprendizaje de las matemáticas básicas, en Colombia. trilogía Ciencia Tecnología Sociedad, 9(16), 63. https://doi.org/10.22430/21457778.182

Carr, M., Taasoobshirazi, G., Stroud, R., \& Royer, J. M. (2011). Combined fluency and cognitive strategies instruction improves mathematics achievement in early elementary school. Contemporary Educational Psychology, 36(4), 323-333. https://doi.org/10.1016/j.cedpsych.2011.04.002

Christie, M., \& de Graaff, E. (2017). The philosophical and pedagogical underpinnings of Active Learning in Engineering Education. European Journal of Engineering Education, 42(1), 5-16. https://doi.org/10.1080/03043797.2016.1254160

Concannon, F., Flynn, A., \& Campbell, M. (2005). What campus-based students think about the quality and benefits of e-learning. British Journal of Educational Technology, 36(3), 501-512. https://doi.org/10.1111/j.1467-8535.2005.00482.x

Del Moral, M. E., Martínez, L. V., \& Neira, M. D. R. (2014). Variables asociadas a la cultura innovadora con TIC en escuelas rurales. Profesorado.

Díaz, J., Schiavoni, A., \& Amadeo, P. (2011). Desarrollo de Objetos de Aprendizaje sobre Accesibilidad Web: un caso de estudio comparando dos Metodologías de Diseño. Proceedings of the 2nd, 60-65. https://www.researchgate.net/profile/Javier_Diaz25/publication/291818224_DE SARROLLO_DE_OBJETOS_DE_APRENDIZAJE_SOBRE_ACCESIBILIDAD_WEB _UN_CASO_DE_ESTUDIO_COMPARANDO_DOS_METODOLOGIAS_DE_DISE NO/links/56a62fa508ae6c437c1ae187.pdf

Duygu, D. C., Alkiş, N., \& Ozkan-Yildirim, S. (2018). A structural model for students' adoption of Learning Management Systems: An empirical investigation in the higher education context. Educational Technology and Society, 21(2), 13-27.

Farha, N. W. (2009). An exploratory study into the efficacy of learning objects. The Journal of Educators Online, 6(2), 1-32.

García Aretio, L. (2005). Objetos de aprendizaje. Características y repositorios. Boletín Electrónico de Noticias de Educación a Distancia (BENED). http://espacio.uned.es/fez/eserv/bibliuned:327/editabril2005.pdf 
García, L. (2013). MOOC: objetos de aprendizaje. Contextos Universitarios Mediados, 19(13), 1-6. $\quad$ http://e-spacio.uned.es/fez/eserv/bibliuned:UNESCOcontextosuniversitariosmediados-13_19/Documento.pdf

Gasparetti, F., De Medio, C., Limongelli, C., Sciarrone, F., \& Temperini, M. (2018). Prerequisites between learning objects: Automatic extraction based on a machine learning approach. Telematics and Informatics, 35(3), 595-610. https:// doi.org/10.1016/j.tele.2017.05.007

Herrera, J. F., Gelvez, N. Y., \& Sánchez, J. M. (2014). Iniciativas de Estandarización en la Producción de Objetos Virtuales de Aprendizaje. Journal of Information Systems and Technology Management, 11(3). https://doi.org/10.4301/s1807$\underline{17752014000300009}$

Hsiung, W. Y. (2018). ID NO. TU015 TOPIC: Humanizing the Online Learning Experience with H5P: A New World of Learning Interactive. UNIVERSITY CARNIVAL on e-LEARNING (IUCEL) 2018, 123.

IEEE. (2011). IEEE Standard for Learning Object Metadata - Corrigendum 1: Corrigenda for 1484.12.1 LOM (Learning Object Metadata). IEEE Std 1484.12.12002/Cor 1-2011 (Corrigendum to IEEE Std 1484.12.1-2002), pp. 1-24. https://doi.org/10.1109/IEEESTD.2011.5982125

Kay, R. H., \& Knaack, L. (2009). Assessing learning, quality and engagement in learning objects: The Learning Object Evaluation Scale for Students (LOES-S). Educational Technology Research and Development, 57(2), 147-168. https://doi.org/10.1007/s11423-008-9094-5

Lau, S. H., \& Woods, P. C. (2008). An investigation of user perceptions and attitudes towards learning objects. British Journal of Educational Technology, 39(4), 685699. https://doi.org/10.1111/j.1467-8535.2007.00770.x

Lavigne, G., Backhoff-Escudero, E., \& Organista-Sandoval, J. (2008). La hibridación digital del proceso educativo.

Marzal, M., Calzado, J., \& Ruvalcaba, E. (2015). Objetos de aprendizaje como recursos educativos en programas de alfabetización en información para una educación superior de posgrado competencial. Investigación bibliotecológica, 29(66), 139168. https:// doi.org/10.1016/j.ibbai.2016.02.029

Mason, R., Weller, M., \& Pegler, C. (2003). Learning in the Connected Economy. Londres.

Morales, L. Y., Gutiérrez, L., \& Ariza, L. M. (2016). Guía para el diseño de objetos virtuales de aprendizaje (OVA). Aplicación al proceso enseñanza-aprendizaje del 
área bajo la curva de cálculo integral. Revista Científica General José María Córdova, 14(18), 127. https://doi.org/10.21830/19006586.46

Naharro, S., Bonet, P., Cáceres, P., Fargueta, F., \& García, E. (2007). Los objetos de aprendizaje como recurso para la docencia universitaria: criterios para su elaboración. En Área de sistema de información y comunicaciones. http://www.aqu.cat/doc/doc_22391979_1.pdf

Navas, E. E., \& Cabero, J. (2007). Objetos de aprendizaje en la Universidad Metropolitana Learning objects in the Metropolitan University. Anales de la Universidad Metropolitana, 8, 121-136.

Rekhari, S., \& Sinnayah, P. (2018). H5P and Innovation in Anatomy and Physiology Teaching. Research and development in higher education: [re] valuing higher education: volume 41: refereed papers from the 41st HERDSA Annual International Conference. 2-5 July 2018, Convention Centre, Adelaide, 41, 191-205. http://vuir.vu.edu.au/37400/

Rosanigo, Z., Bramati, P., López, C., Bramati, S., \& Cotti, L. (2016). TIC y Objetos de Aprendizaje en el Ámbito Educativo. XVIII Workshop de Investigadores en Ciencias de la Computación (WICC 2016), 945-950. http://sedici.unlp.edu.ar/handle/10915/53321

Scott, C. L. (2015). The Futures of Learning 3: what kind of pedagogies for the 21st century? Education Research and Foresight, 1-21.

Singh, S., \& Scholz, K. (2017). Using an e-authoring tool (H5P) to support blended learning: Librarians' experience. Facilitating social learning through learning design: A perspective of collaborative academic development, 158.

Stephenson, J., \& Sangrá, A. (2008). Fundamentos del Diseño Técnico Pedagógico en el E-Learning. Universitat Oberta de Catalunya, 1, 106.

Torrance, B. Y. M., \& Wiggins, C. (2016). What Is xAPI? TD Talent Development, $70(2), 28-31$.

UNESCO. (2015). Guía Básica de Recursos Educativos Abiertos (REA). En Unesdoc.

Vieira, E., De Morales, M., \& Rossato, J. (2016). Evaluation of Virtual Objects: Contributions for the Learning Process. International Review of Research in Open and Distributed Learning, 17(6), 62-74. http://files.eric.ed.gov/fulltext/EJ1122233.pdf

Wiley, D. a. (2007). The Learning Objects Literature. Handbook of Research on Educational Communications and Technology, 16, 345-354. http://opencontent.org/docs/wiley-lo-review-final.pdf 
Williams, J., O'Connor, M., Windle, R., \& Wharrad, H. J. (2015). Using reusable learning objects (rlos) in injection skills teaching: Evaluations from multiple user types. Nurse Education Today, 35(12), 1275-1282. https://doi.org/10.1016/j.nedt.2015.06.001

\section{AUTORES}

\section{Sergio Ramón Rossetti López}

Maestro en Gestión de Tecnologías de la Información e Ingeniero en Sistemas Computacionales. Durante 16 años ha impartido clases sobre informática, estadística, programación, tecnologías de la información y bases de datos en diversas universidades en México. Miembro de la academia de investigaciones organizacionales en la Universidad de Sonora. Miembro de la academia de tecnologías de la información en la Universidad Estatal de Sonora. Estudiante de Doctorado en Innovación en Tecnología Educativa por la Universidad Autónoma de Querétaro y becario del Consejo Nacional de Ciencia y Tecnología (Conacyt).

sergio.rossetti@unison.mx

Orcid ID: https:/ / orcid.org/0000-0002-5524-8674

Google Scholar: https://scholar.google.com/citations?hl=en\&user=Zp-QZksAAAAJ

\section{Ma. Teresa García Ramírez}

Profesora e investigadora en la Facultad de Informática de la Universidad Autónoma de Querétaro, donde ha coordinado proyectos de creación o actualización de programas académicos. Ha dirigido tesis de licenciatura, maestría y doctorado, y ha participado en eventos académicos nacionales e internacionales. Su participación en proyectos le ha permitido publicar sus resultados en eventos y revistas en México, Latinoamérica y Asia. Al igual, ha colaborado como evaluadora de programas académicos nacionales y en comités editoriales de varias revistas nacionales e internacionales.

teregar@uaq.mx

Orcid ID: https:/ / orcid.org/0000-0002-5524-2002

Google Scholar: https://scholar.google.com/citations?user=Fz49LVkAAAAJ\&hl=es

\section{Isaac Shamir Rojas Rodriguez}

Doctor en Desarrollo Regional por el Centro de Investigación en Alimentación y Desarrollo A. C. (CIAD A. C.). Ex becario del Consejo Nacional de Ciencia y Tecnología (Conacyt). Miembro del Sistema Nacional de Investigadores (SNI MÉXICO). Egresado de la Maestría en Calidad de la Universidad La Salle Noroeste. Formado como Ingeniero en Sistemas Computacionales. Actualmente me desempeño como Profesor Investigador del Departamento de Administración de la División de Ciencias Económicas y Administrativas de la Universidad de Sonora. Profesor de las asignaturas. Maestría estadística para la administración, seminario de producción y estudios independientes. Licenciatura; administración de la producción, cultura y gestión organizacional.

shamir.rojas@unison.mx 
Rossetti López, S. R., García Ramírez, M. T. y Rojas Rodríguez, I. S. Evaluación de la implementación de un objeto de aprendizaje desarrollado con tecnología H5P

Orcid ID: https:// orcid.org/0000-0002-0798-1476

Google Scholar: https://scholar.google.com/citations?hl=en\&user=hLdAbk0AAAAI 